\title{
Clinical significance of additional muscular slips in the sole of human cadaver
}

\begin{abstract}
During dissection of a male cadaver of $35-40 \mathrm{yr}$ age, we encounter three variations in intrinsic muscles of sole in layers $1 \& 2$. These muscles influence the action of other muscle but also modify the effect of contact with the ground. An additional muscle was found in first layer bilaterally, abductor ossis metatarsi digit quanta. In same layer, FDB had 3 digitations instead of four, this was observed bilaterally. In second layer flexor hallucis longus (FHL) tendon provided strong slip to flexor digitorum longus (FDL) at knot of Henry, showed digitations for lateral four toes along with digitations of FDL, this was observed in left foot only. The knowledge of variation of foot muscle architecture is utmost importance to orthopedist, radiologist and podiatrist in analysis of foot function, biomechanical modeling of the foot and prosthesis designing. In painful and disabling conditions of foot during differential diagnosis of the presence of accessory muscles of the foot should be kept in mind. On other hand additional muscle slip can be used as replacement flaps in cases of tendon rupture or dysfunction.
\end{abstract}

Keywords: abductor ossis metatarsi digit quanta, flexor digitorum braves, flexor hallucis longus, variation
Volume I Issue 3 - 2015

\section{Richa Niranjan}

Department of Anatomy, Government Medical College, Haldwani, India

\section{Correspondence: Richa Niranjan, Department of Anatomy, Government Medical College, Haldwani, India, Tel 97585I 2594, Email niranjanricha@yahoo.co.in}

Received: October 08, 2015 | Published: October 30, 2015
Abbreviations: FHL, flexor hallucis longus; FDL, flexor digitorum longus; AOMQ, abductor ossis metatarsi quanta; ADM, abductor digit minima

\section{Introduction}

Bipedal locomotion has once again compelled us to go through anatomical aspect of foot in especially to muscle which helps in stabilizing and propagation of foot. Bony configuration remains static but the ligament and muscle play key role in transferring the power to bring the foot in dynamic status. There are 18 intrinsic and 4 extrinsic tendons in the sole of foot. These muscles are subjected to variations which are usually encountered during surgery or cadaveric dissections. As found in literature, accessory muscles like abductor ossis metatarsi quanta muscle and opponent's digit minima muscle can strengthen the parent muscle and used in replacement flaps in tendon injuries ${ }^{1}$ and also have anthropological importance. Flexor digitorum braves subjected to variety of variation absence of digitations to little toe ${ }^{2}$ or supernumerary slip. ${ }^{3}$ There is usually communication between FHL \& FDL muscle. Flexor digitorum accessories might be absent and adductor hallucis, transverse head might be missing. ${ }^{4}$ In our case we found three variations as bilateral presence of abductor ossis metatarsi quanta (AOMQ) muscle and three digitations of FDB, in left foot communicating muscle slip from FHL to FDL.

\section{Case report}

During regular dissection, we found three variations in muscles of sole of foot in a male cadaver 35-40year of age. We found variations in layer $1 \& 2$.

\section{In first layer of sole}

Abductor digit minima arise from both calcanean tuberosity, some fibers arising from lateral process reaches to head of $4^{\text {th }}$ and $5^{\text {th }}$ metatarsal may form separate muscle, Abductor ossis metatarsi digit quanta, receiving nerve supply from lateral plantar nerve (Figure 1) (Figure 2). Abductor of fifth metatarsal in addition to
ADM (abductor digit minima) on tuberosity of fifth metatarsal ${ }^{4}$ or to middle or anterior part of fifth metatarsal, prevalence found to be $40 \% .{ }^{5}$ Our case slip reached up to anterior part of $4^{\text {th }} \& 5^{\text {th }}$ metatarsal. Flexor digitorum braves lies immediately deep to central part of plantar aponeurosis. It arises by a narrow tendon from the medial process of the calcanean tuberosity and intramuscular septa between it and adjacent muscle. It usually divides into 4 tendons for lateral four toes, each slip divide at proximal phalanx and attaches to side of middle phalanx, thus forms tunnel for FDL. In our study we found 3 digitations of FDB, slip to fifth toe was absent (Figure 3) (Figure 4). The slip to given toes may be joined by supernumerary slip or be absent. Nathan and Globe ${ }^{2}$ found the slip to the fifth toe to be most susceptible for such variation (63\%), Bergman et al., ${ }^{6}$ Yalcin and Ozan $^{7}$ and Lobo et al., ${ }^{8}$ Ilayperuma I. ${ }^{9}$ Those to third or fourth less so $(10 \%)$ that to second almost invariable.
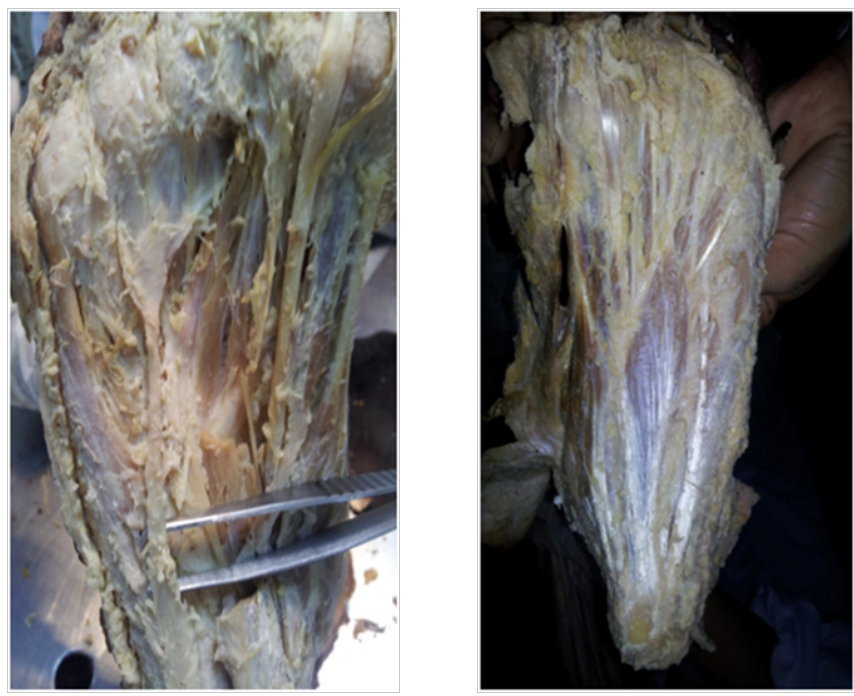

Figure I \& 2 B/L abductor ossis metatarsi digit quanta (marked with arrow). 

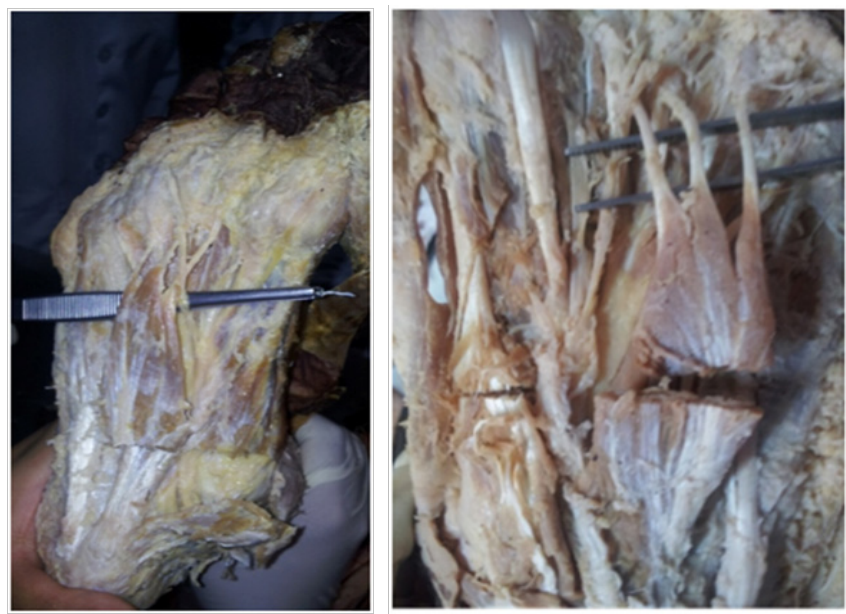

Figure 3 \& 4 B/L 3 digitations of FDB (marked with arrow).

\section{In second layer of sole}

Flexor Hallucis Longus arises from the distal 2/3 of post surface of fibula $\&$ adjoining interosseous membrane. FHL tendon grooves the post surface of lower end of tibia, talus and inferior surface of sustentaculum tail of calcanean. FHL tendon reaches to the interval of sesamoid bone under head of first metatarsal then gets inserted at base of distal phalanx. In sole of foot FHL crosses FDL superiorly from lateral to medial side, at the crossing point (knot of Henry, the long digital flexor receives a strong fibrous slip from FHL tendon. The connecting slip to FDL varies in size, it's usually continues into the tendons for the second \& third toes, but sometime restricted to second and occasionally extends to the fourth. In our study all four of left foot toes received digitations from FHL along with FDL (Figure 5).

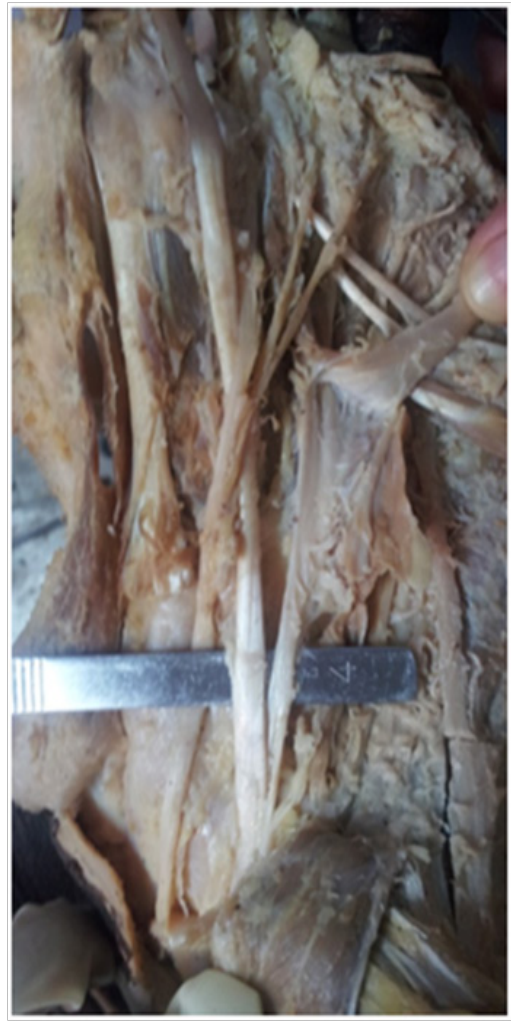

Figure $5 \mathrm{U} / \mathrm{L}$ (Lt side) additional slip of FHL to FDL (at knot of henry).

\section{Discussion}

Human foot has evolved from primarily a grasping organ which was essential for efficient arboreal locomotion and over the course developed to have an elaborate plantar aponeurosis, strong plantar ligaments, longitudinal arches an enlarged muscles flexor accessories, an adducted (non opposable) hallux, a remodeled calcaneocuboid joint, a long tarsus and shortened toes (II to V). ${ }^{10}$ It serves a dual role in bi-pedal locomotion performing as mobile structure and also as a rigid lever. The foot muscles play an important role in positioning of the forces on the foot in both posture and locomotion. ${ }^{11}$ The presence of abductor of fifth metatarsal (abductor ossis metatarsi quanta muscle) can strengthen the parent muscle and can be used as replacement flaps in tendon injuries. ${ }^{1}$ They are part of atavistic group of muscle the 'contravenes' in primates thus have anthropological importance. ${ }^{12}$ These muscles were presented as a muscular sheet in embryo, most of it disappears and only parts of it persist in human foot as rare variation. ${ }^{13}$ For clinical point of view Musculocutaneous flap of FDB is used in reconstruction of heel $\mathrm{pad}^{14-17}$ and tendon transfer is done for correction of flexible claw and hammer toe deformities. ${ }^{18}$ In addition distal plantar area of foot has been reconstructed using FDB muscle flap based on reverse flow lateral plantar artery pedicle. ${ }^{19}$ Normally lateral portion of the FHL gives a tendinous slip to FDL in the sole of foot at knot of Henry. This communication at times may be missing $29 \% .{ }^{20}$ Thus communication between FHL \& FDL have more interaction and influence on each other. ${ }^{21}$ In majority of cases of tibialis posterior dysfunction results in retention of function of both, the hallux and lesser digits due to strong communication. ${ }^{20} \mathrm{FHL}$ tendon can be transferred for reconstruction of chronically ruptured Achilles tendon to be effective, safe and easy surgery in patient with low to moderate demands. ${ }^{22} \mathrm{FHL}$ tendon found to be susceptible for injury at knot of Henry because excessive stress and strain. Patient complains of pain during prolong walking and running, tenderness on palpation at one thumb breadth lateral to tuberosity of navicular. USG and MRI are not found to be helpful in diagnosis. Surgical treatment includes release of knot of Henry, debridement and repair of the longitudinal split in the FHL and excision of the interconnecting tendon between FHL and FDL. ${ }^{23}$ This appears to be additional slip could be remnant of some of the muscles which were involved during process of movement from quadripedal to bipedal locomotion.

\section{Conclusion}

Anatomical variations emphasize us to appreciate the anatomical diversity and variation existing in living organism. It's needed to review these adaptation related to activity of sole. The usage of fifth toe in humans is minimal as compared to little finger, however it plays wider role in maintaining the lateral longitudinal arch. The muscles in relation to lateral longitudinal arch do play in maintaining the stability and static support and finally stabilizing the activity of sole. Therefore it could be presumed that some variation likely to be involved in this region during the process of evolution and mechanism of locomotion. This might be reason behind absence of $4^{\text {th }}$ digitations of Flexor digitorum braves. The knowledge of the anatomical variations of these AOMQ, FDB \& FHL muscle will helpful in surgical procedure of the foot, during reconstructive surgery in case foot injury.

\section{Acknowledgements}

None.

\section{Conflict of interest}

Author declares that there is no conflict of interest. 


\section{References}

1. Pichler W, Tesch NP, Grechenig W, et al. Anatomical Variations of the flexor hallucis longus muscle and consequences for tendon transfer. A cadaver study. Surg Radiol Anat. 2005;27(3):227-231.

2. Nathan H, Globe H. Flexor digitorum brevis-anatomical variations. Anat Anz. 1974;135(3):295-301.

3. Rosse C, Gaddum Rosse P. Hollinshead's Textbook of Anatomy. 5th ed. Philadelphia: Lippincott-Ravenn; 1997.

4. Standring S, Ellis H, Healy JC, et al. Grays Anatomy. 39th ed. New York: Churchill Livingstone; 2005. 1500p.

5. Bergmann RA, Afifi AK, Miyauchi R. Illustrated encyclopedia of human anatomic variation: A Abductor digiti minimi (pedis). 2010.

6. Bergmann RA, Thompson SA, Afifi AK, et al. Compendum of Human Anatomic Variations. Baltimore, USA; 1988.

7. Yalcin B, Ozan H. Some variations of the musculus flexor digitorum brevis. Anat Sci Int. 2005;80(4):189-192.

8. Lobo SW, Menezes RG, Mamata S, et al. Phylogenetic variation in flexor digitorum: A Napalese cadaveric study. Nepal med Coll J. 2008;10(4):230-232.

9. Ilayperuma I. On the variations of muscle flexor digitorum brevis: Anatomical Insight. Int J Morphol. 2012;30(1):337-340.

10. Susman RL. Evolution of human foot: evidence from Plio-Pleistocene hominids. Foot ankle. 1983;3(6):365-376.

11. Williams PL, Bannister LH, Berry MM, et al. Gray's Anatomy: The anatomical basis of medicine and surgery. 38th ed. New York: Churchill Livingstone; 2000.

12. Stark HH, Otter TA, Boyes JH, et al. Atavistic contrahentes digitorum and associated muscle abnormalities of the hand: a cause of symptoms. Report of three cases. J Bone Joint Surg Am. 1979;61(2):286-289.

13. Yamamoto C, Murakami T, Ohtsuka A. Homology of adductor pollicis and contrahentes muscle: A study of monkey hands. Acta Med Okayama. 1988;42(4):215-226.
14. Hartrampf CR, Scheflan M, Bostwick J 3rd. The flexor digitorum brevis muscle island pedicle flap: a new dimension in heel reconstruction. Plast Reconstr Surg. 1980;66(2):264-270.

15. Ikuta Y, Murakami T, Yoshioka K, et al. Reconstruction of the heel pad by flexor digitorum brevis Musculocutaneous flap transfer. Plast Reconstr Surg. 1984;74(1):86-96.

16. Lin SD, Chou CK, Yang C, et al. Reconstruction of plantar heel defect using reinnervated, skin-grafted flexor digitorum brevis flap. Br J Plastic Surg. 1991;44(2):109-112.

17. Stevenson TR, Kling TF, Friedman RJ. Heel reconstruction with flexor digitorum brevis musculocutaneous flap. $J$ pediatr Orthop. $1985 ; 5(6): 713-716$.

18. Tokioka K, Nakatsuka T, Tsuji S, et al. Surgical correction for curly toe using open tenotomy of flexor digitorum brevis tendon. J Plast Reconstr Aesthet Surg. 2007;60(12):1317-1322.

19. Sakai N, Yoshida T, Okumura H. Distal plantar area reconstruction using a flexor digitorum brevis muscle flap with reverse-flow lateral plantar artery. Br J Plastic Surg. 2001;54(2):170-173.

20. Fernanades R, Aguiar R, Trudell D, et al. Tendon in the plantar aspect of foot: MR imaging and anatomic correlation in cadavers. Skeletal Radiol. 2007;36(2):115-122.

21. O’Sullivan E, Carare-Nandi R, Greenslade J, et al. Clinical significance of variations in the interconnections between flexor digitorum longus and flexor hallucis longus in the region of the knot of Henry. Clin Anat. 2005;18(2):121-125.

22. Mahajan RH, Dalal RB. Flexor hallucis longus tendon transfer for reconstruction of chronically ruptured Achilles tendon. J Orthopn Surg (Hong kong). 2009;17(2):194-198.

23. Boruta PM, Beauperthuy GD. Partial tear of the flexor hallucis longus at knot of Henry: presentation of three cases. Foot Ankle Int. 1997;18(4):243-246. 